http://jmscr.igmpublication.org/home/

ISSN (e)-2347-176x ISSN (p) 2455-0450

crossref DOI: https://dx.doi.org/10.18535/jmscr/v7i12.47

Journal Of Medical Science And Clinical Research

IGM Publication

An Official Publication of IGM Publication

\title{
Pattern of antibiotic sensitivity to microorganism isolated from patient presenting with ear discharge
}

\author{
Authors \\ Neelikattu Aathira Tess Thomas, Kalpana Rajiv Kumar, Swapnil Gosavi, \\ Jubina Puthen Purayil, Apurva Sharma \\ Corresponding Author \\ Kalpana Rajiv Kumar
}

\begin{abstract}
Long standing ear infection is very prevalent in developing countries. Appropriate treatment of Chronic otitis media is of utmost importance to avoid various longstanding health issues, including hearing impairment and deficit in social communication.

Purpose of the study: To isolate the causative bacteria and determine the antibiotic sensitivity for the same.

Material and Method: In this study, we included 30 patients that presented to our ENT OPD with complaints of ear discharge. Swab from discharging ear were collected and sent for aerobic culture and susceptibility testing.

Conclusion: Of the total bacterial swabs the organism isolated predominantly were Pseudomonas aeruginosa, followed by Staphylococcus aureus and Proteus spp.

Amongst Gram Positive bacteria, highest resistance was seen for penicillin 10(76\%), followed by tetracycline and ampicillin 9(69.2\%). Whereas sensitivity was found to amoxicillin in 12(92\%), ciprofloxacin in 10(76.9\%), azithromycin in 9(69.3\%) and Co-trimazole in 8(61.5\%). P. aeruginosa showed highest resistance to amoxicillin 15(88.2\%) followed by ampicillin 12(70.5\%) and showed highest sensitivity to ciprofloxacin 12(70.5\%), followed by 10(58.8\%) for amikacin and gentamycin. Based on these findings, guided approach for treatment according to the antibiotic sensitivity is necessary.
\end{abstract}

\section{Introduction}

Otitis media is one of the leading causes affecting all age groups equally. It increases the number of OPD visits as well as economic burden over the patients and the healthcare system in developing countries $^{(1)}$.

According to an estimate by WHO, people with hearing impairment resulting due to otitis media has increased from 42 million to 250 million in the last 16 years, with approximately three quarters of the individual acquiring adult onset hearing loss ${ }^{(6)}$.

Otitis media is characterized by an inflammatory process of the middle ear cleft. Various anatomical and immunological factors along with crowded living condition and malnutrition results in a significant prevalence of patients suffering from otitis media with varying degree of severity $^{(2)}$. 
Adult population is equally affected by otitis media even though it is considered to be primarily affecting the younger population, ${ }^{(3)}$ the disease begins in the childhood or as a sequel to inappropriate management of the acute suppurative otitis media ${ }^{(4)}$.

Undertreated or inappropriate management of the condition leads to purulent otitis media, often with perforation and further complications including recurrent acute otitis media, persistence of middle ear effusion leading to hearing impairment, it may also complicate into mastoiditis, meningitis, brain abscess and sepsis ${ }^{(5)}$.

Many studies have indicated that, Staphylococcus aureus, Pseudomonas aeruginosa, Proteus mirabilis, Klebsiella pneumonia and Escherichia coli are the commonest organisms to be isolated from ear swabs ${ }^{(5,7,8)}$

Treatment of ear infection is not always apt since drug susceptibility patterns change overtime and empirical antibiotic therapy may not be effective at times and could contribute to development of antimicrobial resistance ${ }^{(9,10)}$.

Prompt and effective treatment of ear infection will significantly reduce both short and long term complications associated with ear infection and can also improve the quality of life of patients with ear infection.

There has been an increase in antibiotic resistance noted over a period of time due to irrational use of antibiotics, however there has been limited research evidence available about the same. Hence this study has been undertaken, to determine the causative bacterial flora responsible for otitis media and its antibiotic sensitivity pattern.

\section{Materials and Methods}

A prospective randomized study included 30 patients presenting to the Outpatient department of our hospital with ear discharge.

\section{Inclusion Criteria}

1) Patients more than 5years of age.

2) Patient presenting with activetubo tympanic COM

\section{Exclusion Criteria}

1) Patients who were on topical/systemic antibiotics.

2) Patients with suspected or diagnosed malignancy.

3) Patient presenting with otomycosis, atticoantral COM and otitis externa.

4) Acute suppurative otitis media.

The ear swab specimens of all the subjectswere collected under aseptic precautions and analysed for microbial testing. Care was taken to avoid surface contamination (the pinna was pulled outward and backward to make the external auditory canal more straight to take the ear swab, then sterile cotton swabs were gently rotated in the ear discharge and taken out). All organisms grown were identified according to the standard microbiological methods by using Gram staining reaction, culture characters, colony morphology, pigment production, and type of hemolysis on blood agar.

\section{Results and Observations}

In the present study, individuals ranged from the age group of 10 to 73 years. The highest percentage $10(33.3 \%)$ of ear infection was found among subjects ranging from $10-20$ years.

\begin{tabular}{|l|c|c|}
\hline AGE & $\begin{array}{c}\text { EAR INFECTION } \\
(\mathbf{n = 3 0})\end{array}$ & PERCENTAGE \\
\hline $10-20$ & 10 & $33.3 \%$ \\
\hline $21-30$ & 9 & $30 \%$ \\
\hline $31-40$ & 3 & $10 \%$ \\
\hline $41-50$ & 2 & $6.6 \%$ \\
\hline $51-60$ & 3 & $10 \%$ \\
\hline $61-70$ & 2 & $6.6 \%$ \\
\hline $\begin{array}{l}\text { Above } \\
70 \text { years }\end{array}$ & 1 & $3.33 \%$ \\
\hline
\end{tabular}

The proportion of ear infection was higher in males $22(73 \%)$ as compared to females who were $8(26.6 \%)$.

Table 1: Sex Distribution amongst subjects presenting with ear discharge

\begin{tabular}{|l|c|c|}
\hline SEX & $\begin{array}{c}\text { EAR INFECTION } \\
(\mathbf{n}=\mathbf{3 0})\end{array}$ & PERCENTAGE \\
\hline MALE & 22 & $73 \%$ \\
\hline FEMALE & 8 & $26.6 \%$ \\
\hline
\end{tabular}


Out of all the positive swabs, 21(70\%) demonstrated single organism and 9(30\%) were positive for 2 organisms.

Amongst the individuals, Gram Negative bacteria were $17(56.4 \%)$ and $13(43.6 \%)$ were Gram Positive.

Graph 1: Distribution of subjects according to Gram Positive and Gram Negative isolates.

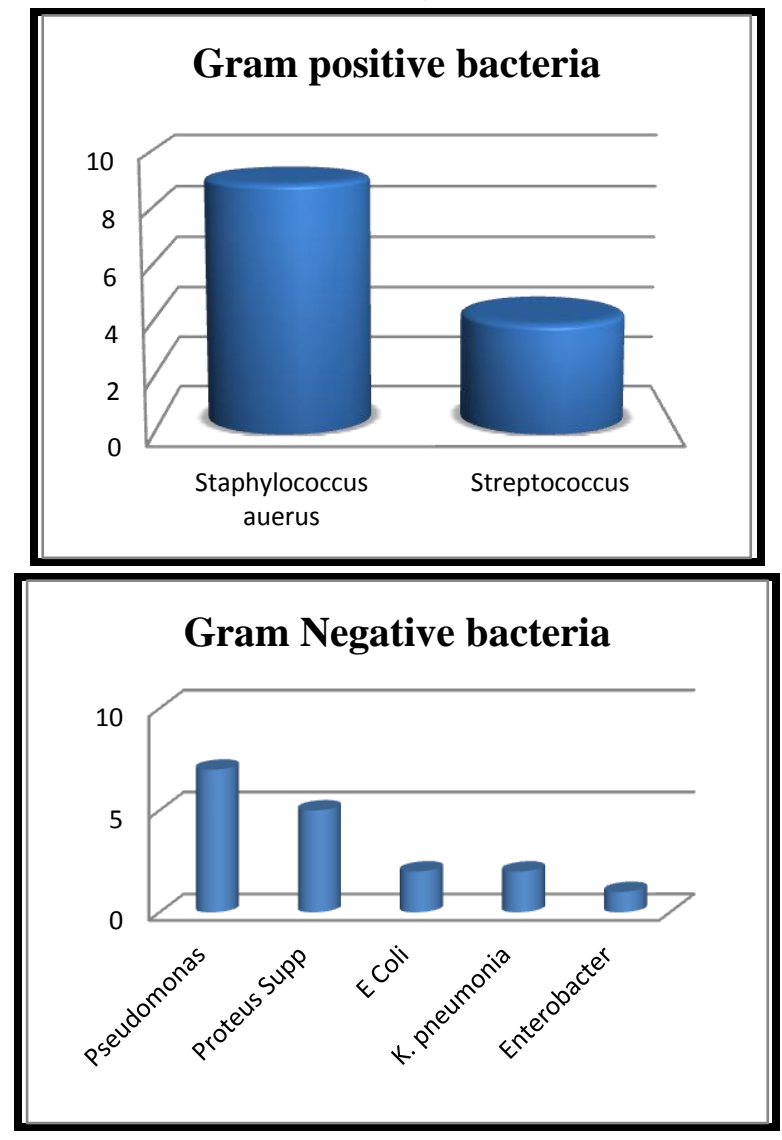

In our study Gram Positive bacteria (Staph auerus, strep) showed highest resistance to penicillin $10(76 \%)$, followed by tetracycline and ampicillin 9(69.2\%). Sensitivity was found to amoxicillin in 12(92\%), ciprofloxacin in 10(76.9\%), azithromycin in $9(69.3 \%)$ and Co-trimazole in $8(61.5 \%)($ Tab 1$)$.

Table 1: Antimicrobial resistance patterns of Gram Positive bacterial isolates $(n=13)$ from ear swab taken from study subjects.

\begin{tabular}{|l|c|c|}
\hline \multirow{2}{*}{ ANTIBIOTICS } & \multicolumn{2}{|c|}{$\begin{array}{c}\text { Gram Positive Bacteria } \\
(\mathrm{n}=13)\end{array}$} \\
\cline { 2 - 3 } & Resistant (\%) & Sensitive (\%) \\
\hline $\mathrm{P}$ & $10(76 \%)$ & $3(23 \%)$ \\
\hline TE & $9(69.2 \%)$ & $4(30.7 \%)$ \\
\hline AMC & $1(7.6 \%)$ & $12(92 \%)$ \\
\hline
\end{tabular}

\begin{tabular}{|l|c|c|}
\hline CF & $3(23 \%)$ & $10(76.9 \%)$ \\
\hline AZM & $4(30.7 \%)$ & $9(69.3 \%)$ \\
\hline AMP & $9(69.2 \%)$ & $4(30.7 \%)$ \\
\hline COT & $5(38.4 \%)$ & $8(61.5 \%)$ \\
\hline
\end{tabular}

KEY: (P)penicillin, (TE)tetracycline, (AMC) amoxicillin/ clavulanicacid, (CF)ciprofloxacin, (AZM) azithromycin, (AMP)ampicillin, (COT)co trimazole.

In our study, P. aeruginosa, Proteus supp, K. pneumonia, E.coli and Enterbacter were resistant to amoxicillin $15(88.2 \%)$ and ampicillin 12 (70.5\%), whereas ceftriaxone 9 (52.9\%), gentamycin10(58.8\%), amikacin10(58.8\%), and ciprofloxacin $12(70.5 \%)$ were effective against Gram Negative bacteria (Tab 2).

Table 2: Antimicrobial resistance patterns of Gram Negative bacterial isolates $(n=17)$ from ear swab taken from study subjects

\begin{tabular}{|c|c|c|}
\hline \multirow{2}{*}{ ANTIBIOTICS } & \multicolumn{2}{|c|}{$\begin{array}{c}\text { Gram Negative Bacteria } \\
(\mathrm{n}=17)\end{array}$} \\
\cline { 2 - 3 } & Resistant $(\%)$ & Sensitive (\%) \\
\hline AMC & $15(88.2 \%)$ & $2(11.7 \%)$ \\
\hline CF & $5(29.4 \%)$ & $12(70.5 \%)$ \\
\hline G & $7(41.1 \%)$ & $10(58.8 \%)$ \\
\hline AMP & $12(70.5 \%)$ & $5(29.4 \%)$ \\
\hline AK & $7(41.1 \%)$ & $10(58.8 \%)$ \\
\hline CTR & $8(47 \%)$ & $9(52.9 \%)$ \\
\hline
\end{tabular}

Key: (AMC) amoxicillin/clavulanic acid, (CF)ciprofloxacin, (G) gentamycin, (AMP)ampicillin, (AK)amikacin, (CTR) ceftriaxone.

\section{Discussion}

Otologist all around the world are observing a high rise in antibiotic resistance amongst people presenting with ear discharge. This rise has been attributed to excessive consumption of untargeted antibiotics, as well as Biofilm formation which is emerging as a factor for persistence of the infection. Early ear swab bacteriological diagnosis helps prescription of appropriate antibiotics, assessment of bacterial resistance, safety, risk of toxicity of the prescribed antibiotics.

- In our study the maximum number of subjects who presented with ear discharge was amongst the age group of 10-20 years. Hailu D et al. ${ }^{(11)}$ in their study found the highest proportion of ear infection among patients in the age group of 1- 20 years. 
- In the present study, a higher preponderance of ear infection was found in males as compared to females. These finding were similar to Muluye et al. ${ }^{(12)}$.

- In our study, Gram Negative bacteria were $17(56.4 \%)$ of which P. aeruginosa was the commonest isolate $7(41.1 \%)$, followed by Proteus spp. 4(23.5\%). Whereas Staph auerus $9(69.2 \%)$ found to be the commonest isolate amongst Gram Positive bacteria $13(43.3 \%)$, followed by Streptococcous 4(30.7\%). Gaur RS et al. ${ }^{(13)}$ had similar findings in their study which revealed that P. aeruginosa (30.96\%) was the most common isolate in COM followed by $\mathrm{S}$. aureus $(29.65 \%)$. The prevalence of $\mathrm{P}$. aeruginosa could be due tothe organisms ability to produce enzymes like proteases to stop the normal defense mechanism of body required to fight infections ${ }^{(14)}$

- In the present study, Gram Positive bacteria (Staph auerus, strep) showed highest resistance to penicillin 10(76\%), followed by tetracycline $9(69 \%)$ and ampicillin $9(69.2 \%)$. This was supported by findings in the study by Abera et al. ${ }^{(15)}$. Whereas they exhibit sensitivity to amoxicillin in $12(92 \%)$, ciprofloxacin in 10(76.9\%), azithromycin in $9(69.3 \%)$ and Co-trimazole in $8(61.5 \%)$.

- In our study, P. aeruginosa showed highest resistance to amoxicillin 15(88.2\%) followed by ampicillin 12(70.5\%), which were similar to the findings in the study conducted by Aberaet $\mathrm{al}^{(15)}$. However $\mathrm{P}$. aeruginosa showed highest sensitivity to ciprofloxacin $12(70.5 \%)$, and $10(58.8 \%$ ) for amikacin and gentamycin. Gaur RS et al. ${ }^{(13)}$ in their study noted $62.5 \%$ of isolates to be susceptible to Ciprofloxacin. Whereas Jang CH et al. ${ }^{(16)}$ carried out a study in 2004 in which Ciprofloxacin resistance was noted in $100 \%$ of the isolates.

\section{Conclusion}

According to the findings of our study, $P$. aeruginosa was the most common isolate followed by S. aureus, and Proteus spp. P. aeruginosa showed highest susceptibility to ciprofloxacin $70.5 \%$, followed by amikacin and gentamycin $58.8 \%$. S. aureus showed highest sensitivity to amoxicillin $92 \%$ followed by ciprofloxacin $76.9 \%$. Hence based on the results of our study, treatment of otitis media through a guided approach according to antibiotic susceptibility of bacterial isolates is recommended.

\section{Reference}

1. Monasta L, et al. Burden of disease caused by otitis media: systematic review and global estimates. PLoS One. 2012;7 (4):e36226.

2. Cripps AW, Kyd J. Bacterial otitis media: current vaccine development strategies. Immunol Cell Biol. 2003;81(1):46-51.

3. Farhan EA, Pooja KK, Najla AA, Anum DA, Geeta B. Ear infections in Karachi: the frequency and antibiotic resistance of bacterial isolates. Pak J Med Sci. 2011;27(1):177-181.

4. Afolabi OA, Salaudeen AG, Ologe FE, Nwabuisi C, Nwawolo CC. Pattern of bacterial isolates in the middle ear discharge of patients with chronic suppurative otitis media in a tertiary hospital in North central Nigeria. African Health Science 2012;12(3):362-7.

5. Seid A, et al. Bacterial otitis media in all age group of patients seen at Dessie referral hospital, north East Ethiopia. Egyptian Journal of Ear, Nose, Throat and Allied Sciences. 2013;14(2):73-8.

6. Smith A, Mathers C. Epidemiology of infection as a cause of hearing impairment. Newton VE, Vallely PJ (Editors).Infection and hearing impairment. John Wiley \& Sons Ltd, England 2006: 31-36.

7. Abera B, Kibret M. Bacteriology and antimicrobial susceptibility of otitis media 
at dessie regional health research laboratory, Ethiopia. Ethiop J Health Dev 2011;25(2):162-167.

8. Muluye D, Wondimeneh $\mathrm{Y}$, Ferede G, Moges F, Nega T. Bacterial isolates and drug susceptibility patterns of ear discharge from patients with ear infection at Gondar University Hospital, Northwest Ethiopia. BMC Ear Nose Throat Disord 2013;13(1):10.

9. Grevers G. Challenges in reducing the burden of otitis media disease: an ENT perspective on improving management and prospects for prevention. Int $\mathrm{J}$ Pediatr Otorhinolaryngol. 2010;74(6):572-7.

10. Agrawal A, et al. Microbiological profile and their antimicrobial sensitivity pattern in patients of otitis media with ear discharge. Indian Journal of Otology. 2013;19(1):5.

11. Hailu D, Mekonnen D, Derbie A, Mulu W, Abera B. Pathogenic bacteria profile and antimicrobial susceptibility patterns of ear infection at Bahir Dar Regional Health Research Laboratory Center, Ethiopia. Springerplus 2016; 5: 466.

12. Muluye D, Wondimeneh Y, Ferede G, Moges F, Nega T. Bacterial isolates and drug susceptibility patterns of ear discharge from patients with ear infection at Gondar University Hospital, Northwest Ethiopia. BMC Ear Nose Throat Disord 2013 Aug 6; 13(1):10.

13. Gaur RS, Mathew J, Varghese AM, Mathew GA, Chandrasekharan R, Anandan S. Microbiological pattern of ear swabs in chronically discharging ears in a Tertiary Care hospital in India. Indian $\mathbf{J}$ Otol 2013;19:51-4.
14. Seid A, Deribe F, Ali K, Kibru G. Bacterial otitis media in all age group of patients seen at Dessie referral hospital, North East Ethiopia. Egypt J Ear Nose Throat Allied Sci2013;14:73-78.

15. Abera B, Kibret M. Bacteriology and antimicrobial susceptibility of otitis media at dessie regional health research laboratory, Ethiopia. Ethiop J Health Dev. 2011;25(2):162-167.

16. Jang CH, Park SY. Emergence of ciprofloxacin-resistant pseudomonas in chronic suppurative otitis media. Clin Otolaryngol Allied Sci 2004;29:321-3. 